\title{
Experimental Results of Converted NAH Method*
}

\author{
Masao NAGAMATSU**
}

\begin{abstract}
Among sound source localization methods, the acoustic intensity (AI) method is widely used in industry. On the other hand, nearfield acoustic holography (NAH) method, which is also a localization method, has not spread to industry use because of its difficulty in measuring high frequency measurement. For high frequencies, the NAH method requires a very large number of measurement points, which results in a prolonged measurement time and very large size. I have proposed a theoretically converted NAH method $^{(1),(2)}$, which uses the extra spatial-domain spectrums in the backpropagating computation of NAH method. This method can measure the sound field using a reasonable number of measurement points even for high frequencies. As a result, the workload of measurement is reduced to the same level as that using acoustic intensity method. In this paper, the theory of the proposed method is explained, and some experimental results are presented to verify this method.
\end{abstract}

Key Words: NAH, Acoustic Holography, Localization, Extra Spectrum, Acoustic Intensity

\section{Introduction}

Recently, mechanical products have become highly sophisticated, and their various characteristic requirements have become more demanding. The requirement about noise problems have also stricter. Therefore, for mechanical products, more precise noise measurement is needed at the development stage.

In the development of mechanical products, AI method is widely used for noise source localization, because the AI method is quite useful, and the AI measurement system is commercially available. On the other hand, there is another sources localization method, the holography method. However it has not spread to industry use.

Compared with the AI method, which gives a physical value from the acoustic pressures measured by a pair of microphones, holography method uses backpropagation computation to reconstruct the sound field. Therefore, holography methods can obtain a sharper image of the sound source distribution than the AI method. There are two main holography methods. One is the acoustic holography (AH) method which measures from far field. Another is the NAH method which measures a near field, the same as in $\mathrm{AI}^{(3),(4)}$. The reason why NAH has not spread is mainly the problem in measurement. In NAH, a two-

\footnotetext{
* Received 16th January, 2006 (No. 05-4282)

** Department of Mechanical Systems Engineering, Hokkaido Institute of Technology, 15-4-1 Maeda-7-jou, Teine-ku, Sapporo-shi, Hokkaido 006-8585, Japan.

E-mail: nagamatu@ hit.ac.jp
}

dimensional spatial-domain Fourier transform is applied to the measured data; therefore, the measurement of the sound field must obey the sampling law. In the measurement of a high-frequency sound field, soundwavelength becomes short, and measurement must be performed with shorter a spatial sampling pitch. This problem has occur since the initial development of NAH, although a method of combining NAH and a beam-forming method to overcome this disadvantage has been published ${ }^{(5)}$.

I propose theoretically converted NAH, which uses the extra spectrums in the spatial-domain Fourier transform. This method can reduce the number of measurement points, therefore, it can overcome the aforementioned disadvantage. Using this method, the workload of the measurement of a sound field is reduced to almost the same level to measurement using AI.

\section{Theory of Converted NAH Method}

The theory of the proposed method is the same as the conventional NAH theory, except for the spatial-domain Fourier transform and inverse transform. Between the Fourier transform and the inverse Fourier transform, this method uses extra spatial-domain spectrums, which are not normally used. In Fourier transform theory, the maximum number of spectrums is the number of data values used for the computation, even when ignoring the aliasing error. However in the proposing method, spectrums exceeding these limits are used. These spectrums are calculated by a spatial domain Fourier transform and involved in spatial-domain inverse Fourier transform. 
The equations of the two-dimensional spatial-domain Fourier transform are:

$$
\begin{aligned}
& \hat{p}\left(k_{x}, k_{y}\right)=\frac{d^{2}}{2 \pi} \sum_{x, y} p(x, y) e^{-i\left(x k_{x}+y k_{y}\right)} \\
& k_{x}=\frac{2 \pi}{d m} b \\
& k_{y}=\frac{2 \pi}{d m} c
\end{aligned}
$$

where $p$ is the measured complex sound pressure value, $P$ is the spatial-domain spectrum, $k_{x}$ and $k_{y}$ are the spatialdomain frequencies, $x$ and $y$ are the actual spatial location of the measurement point, $b$ and $c$ are integral values and $m$ is the number of measurement points along each axis. In conventional $\mathrm{NAH}, b$ and $c$ are defined as:

$$
b, c=-\frac{m-1}{2}, \cdots, 0, \cdots, \frac{m-1}{2},
$$

In the proposed method, these numbers are increased without increasing the number of sampled datum $P$, as follows:

$$
b, c=-\frac{m-1}{2}-n, \cdots, 0, \cdots, \frac{m-1}{2}+n,
$$

where $n$ is the number of extra spatial-domain spectrums at low and high frequencies. The actual increase in the number of spectrums in the calculation is $2 n$. By increasing this number in the Eq. (1), redundant spectrums, "the extra spectrums" in spatial-domain are produced.

Finally, the half-free-space Green function is multiplied and an inverse Fourier transform is performed as shown by Eq. (6). In conventional NAH, the spectrums produced using Eq. (4) are involved in the summation of Eq. (6). In the proposed method, the reconstructed image is produced by using Eq. (6) which involves the extra spectrums in summation.

$$
r(x, y)=\frac{1}{2 \pi} \sum_{k_{x}, k_{y}} \hat{p}\left(k_{x}, k_{y}\right) G\left(k_{x}, k_{y}, z\right) e^{i\left(x k_{x}+y k_{y}\right)}
$$

$G$ is the half free space Green function,

$$
G\left(k_{x}, k_{y}, z\right)=e^{i z \sqrt{k^{2}-k_{x}^{2}-k_{y}^{2}}} .
$$

Using extra spectrums, the proposed method can overcome the shortage of measurement points, and the shortage of spatial sampling number. According to the sampling law of Fourier transform, the sampling pitch must be lower than half the wavelength. This is also applicable to the spatial-domain Fourier transform. In a $1 \mathrm{kHz}$ sound wave, the half-wavelength is about $17 \mathrm{~cm}$, but in $10 \mathrm{kHz}$, it is about $1.7 \mathrm{~cm}$. To apply the sampling laws, the spatial measurement pitch on the measurement plane is shorter, and the number of measurement points on the measurement plane becomes very large. However, by using extra spectrums, the number of spatial-domain spectrums can be increased. Therefore, the very large number of spectrums, which are required by the sampling law can be obtained by low-pitch sampling. In other words, if the experiment is executed with few measurement points, a sufficient number of spatial-domain spectrums to apply NAH can be obtained using the proposed method. In conventional NAH, the pitch between measurement points is usually set at slightly longer than half the wavelength. In the proposed method, the pitch between measurement points can be extended to longer than half wavelength. In this case, the total number of spatial-domain spectrums, which includes the original spectrums and the extra spectrums, should be adjusted to the number that the conventional method would acquire. By modifying the number of spectrums to the number the sampling law, the proposed NAH acts the localization, although there is some deterioration in the reconstructed image.

\section{Experimental Equipment}

The experimental equipment contains at least two microphones. One microphone is the scan microphone, which scans the two-dimensional measurement plane on the sound field. Another is the reference microphone, which is located at a fixed position.

In AI measurement, two microphones are used. These two microphones scan the sound field. In NAH, two mi-

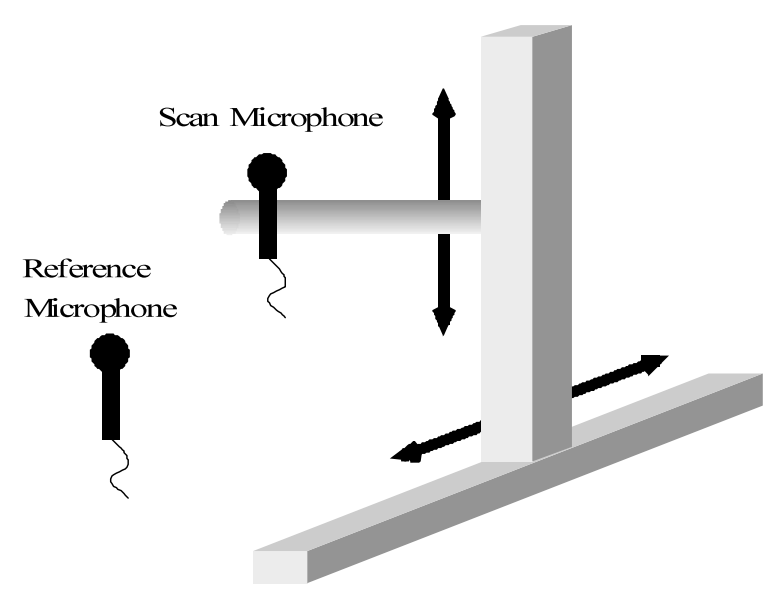

Fig. 1 NAH measurement equipment

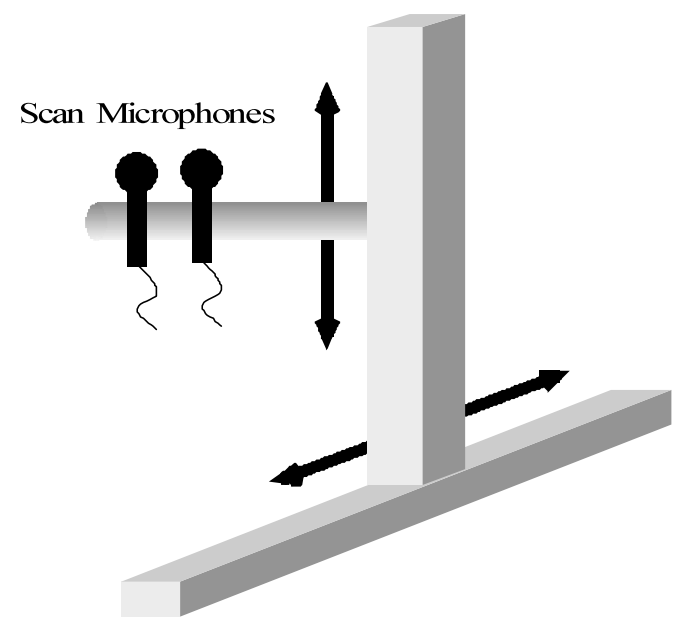

Fig. 2 AI measurement equipment 
crophones are also used. In conventional NAH, the number of measurement point is very large in high-frequency measurement. However, by using the proposed method, the number of measurement points can be reduced to the same level as that when using AI. Therefore, the equipment for two (AI and proposed) methods are exactly the same, except for the location of one microphone.

Figure 1 shows the equipment used for NAH measurement, which consists of two microphones and a microphone traverse mechanism.

Figure 2 shows the equipment used for AI. The two microphones are usually packaged as an intensity probe.

Figure 3 shows the measurement equipment used in the experiments. To measure the sound field for both NAH and $\mathrm{AI}$ methods, this equipment contains 3 microphones.

\section{Experimental Results}

Experiments are performed to verify the proposed method. In the experiments, the sound sources used are

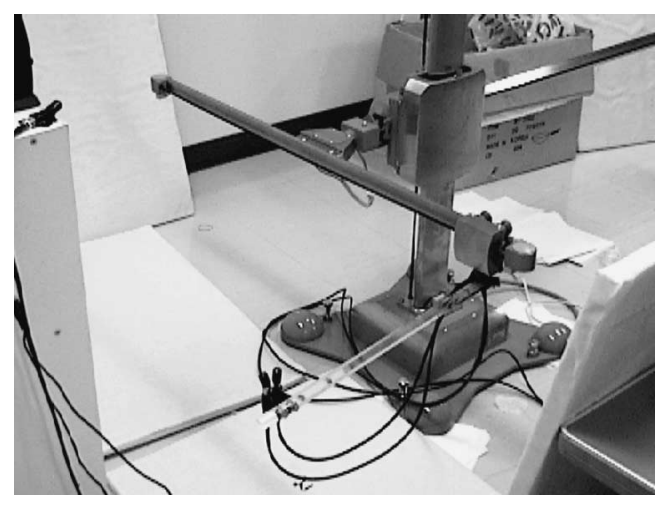

Fig. 3 The measurement equipment used

Table 1 Parameters of experiments

\begin{tabular}{|l|c|c|c|c|}
\hline Experiment No. & 1 & 2 & 3 & 4 \\
\hline Sound frequency & \multicolumn{3}{|c|}{$10 \mathrm{kHz}$} \\
\hline Measurement distance & \multicolumn{3}{|c|}{$0.08 \mathrm{~m}$} \\
\hline Size of measurement plane & \multicolumn{3}{|c|}{$(\mathrm{X}) 1 \mathrm{~m}(\mathrm{Y}) 1 \mathrm{~m}$} \\
\hline Pitch of measurement points & $(\mathrm{X}) 0.017 \mathrm{~m}(\mathrm{Y}) 0.017 \mathrm{~m}$ & \multicolumn{2}{|c|}{$(\mathrm{X}) 0.1 \mathrm{~m}(\mathrm{Y}) 0.1 \mathrm{~m}$} \\
\hline Reconstruction theory & \multicolumn{2}{|c|}{ Normal NAH } & Converted NAH & \\
\hline Number of spatial spectrums & $(\mathrm{X}) 59(\mathrm{Y}) 59$ & $(\mathrm{X}) 11(\mathrm{Y}) 11$ & $(\mathrm{X}) 59(\mathrm{Y}) 59$ & \\
\hline
\end{tabular}

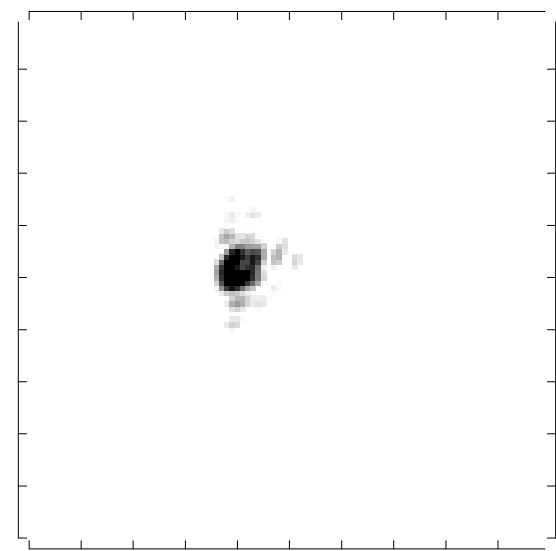

Fig. 4 Experimental result 1 for single source one or two small speakers. Three sets of experiments are performed. One set of experiments is performed using one speaker, and the other sets are performed using two speakers with a phase difference set at $0 \mathrm{deg}$ or $180 \mathrm{deg}$.

In each set of experiments, the following are tested. The use of NAH for high-density measurement that satisfies the sampling law, the use of NAH for low-density measurement that does not satisfy the sampling law, the

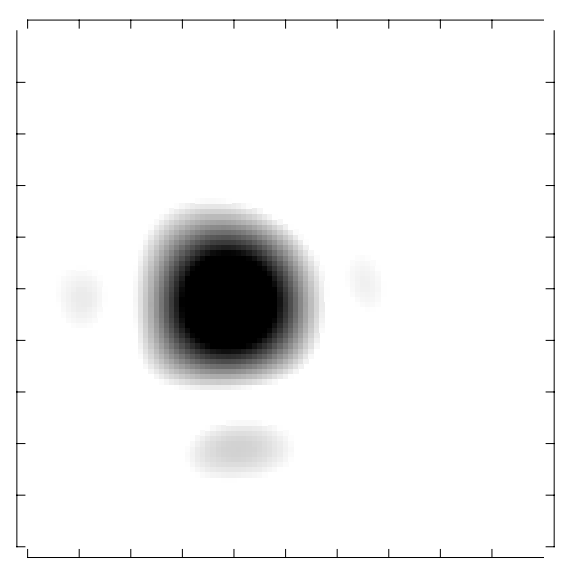

Fig. 5 Experimental result 2 for single source

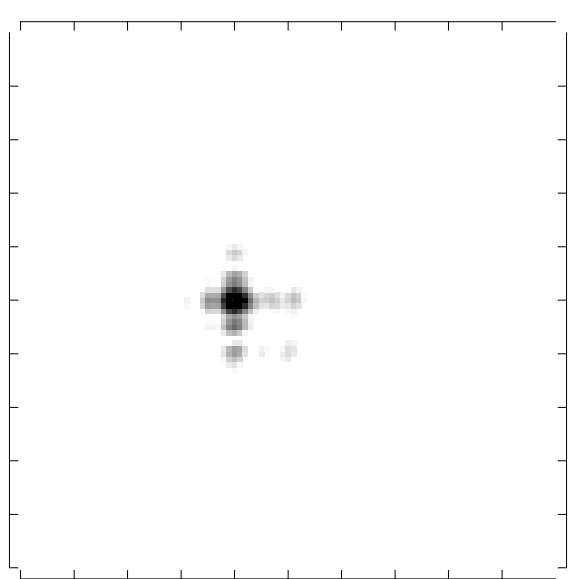

Fig. 6 Experimental result 3 for single source

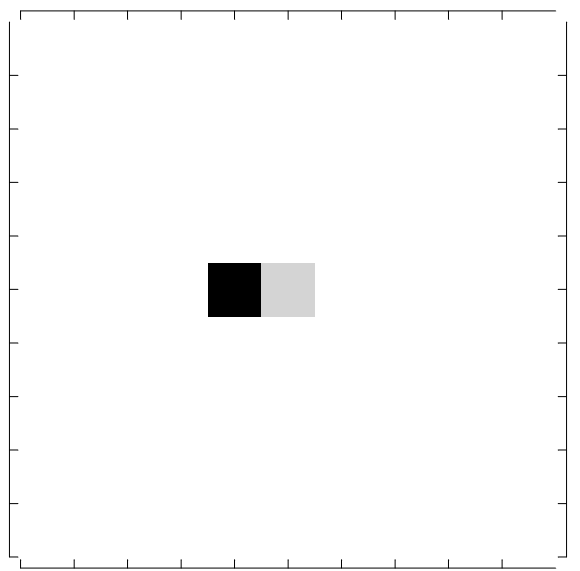

Fig. 7 Experimental result 4 for single source 


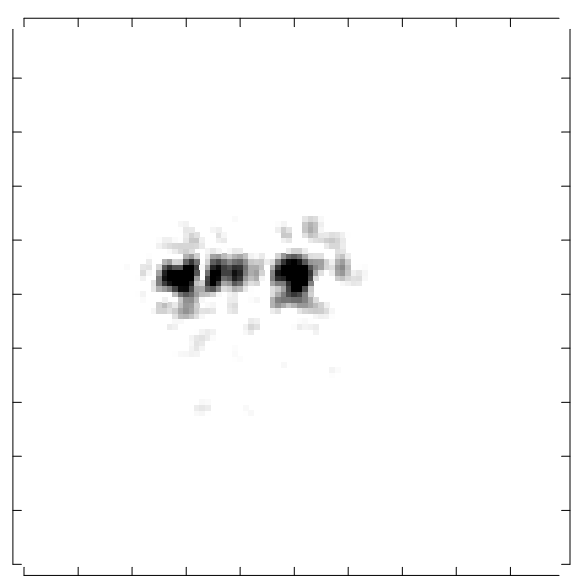

Fig. 8 Experimental result 1 for same-phase sources

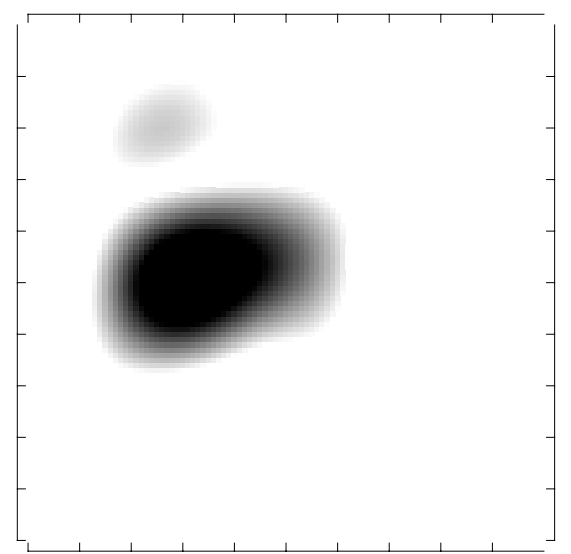

Fig. 9 Experimental result 2 for same-phase sources

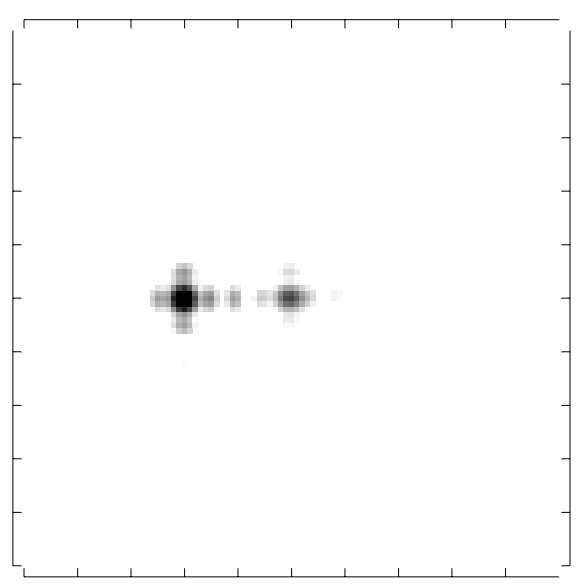

Fig. 10 Experimental result 3 for same-phase sources

use of the proposed NAH with extra spectrums for lowdensity measurement, and the use of AI for low-density measurement for comparison.

Table 1 shows the measurement parameters of each set of experiments.

The results of the experiments for the single sound source are as follows.

Experimental result 3, which is the result of the proposed method, shows a quite sharp image of the sound

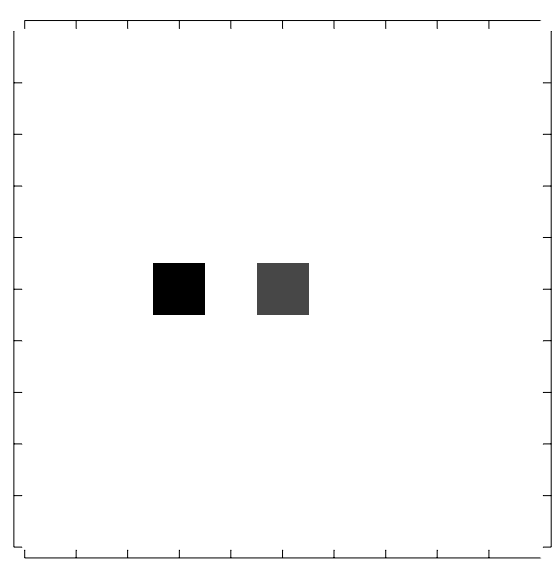

Fig. 11 Experimental result 4 for same-phase sources

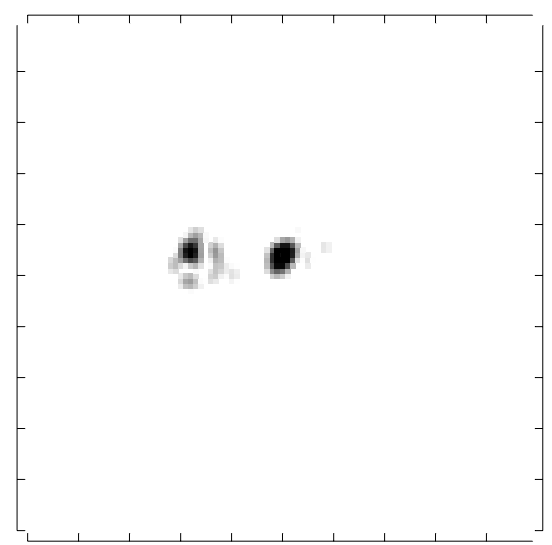

Fig. 12 Experimental result 1 for opposite-phase sources

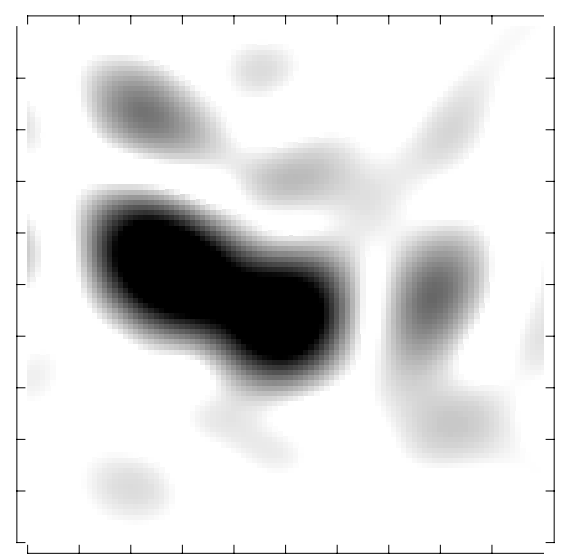

Fig. 13 Experimental result 2 for opposite-phase sources

source similar to that in experimental result 1 , the conventional NAH result, which requires a very large number of measurement points. The AI result shows a large image of the sound source, because the AI computation is for a "single point", without using backpropagation; thus, there is no interpolation of the image.

The workloads of measurement in the cases of Figs. 6 and 7 are absolutely the same. The only difference is the location of the microphones. However, the difference in resolution is clear. Compared with the result shown in 


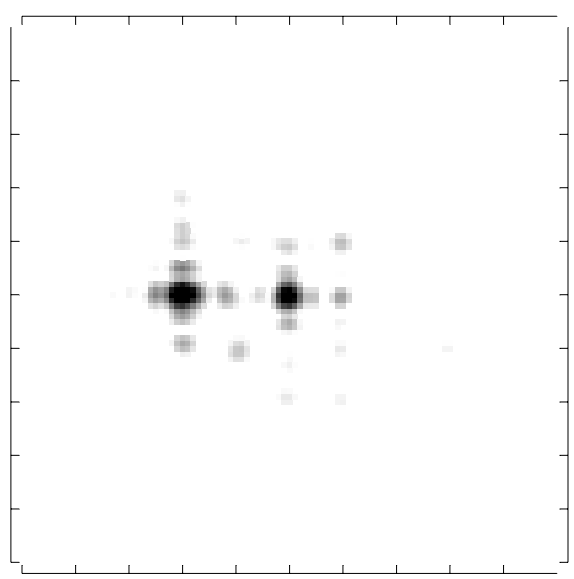

Fig. 14 Experimental result 3 for opposite-phase sources

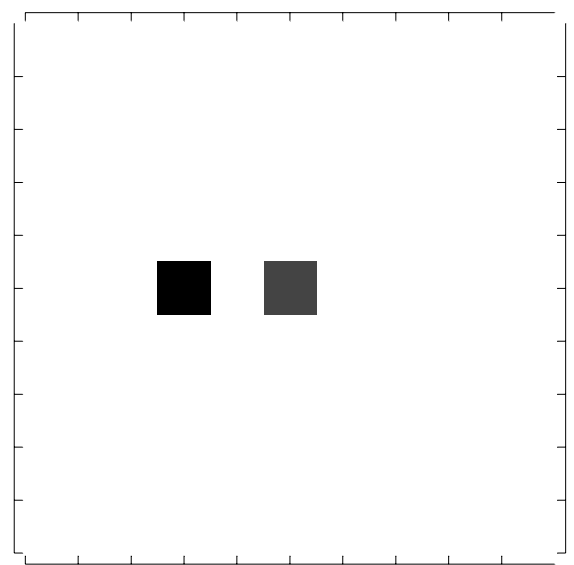

Fig. 15 Experimental result 4 for opposite-phase sources

Fig. 4 which takes over 80 min for measurement, the result shown in Fig. 6 takes only $8 \mathrm{~min}$.

Figures 8-12 show the results for two sound sources of the same phase.

Finally, the results for two sound sources with 180 deg phase difference are shown in Figs. 12-15.
Both sets of experimental results show the same tendency as observed in experiments using a single sound source. Therefore it is verified that the proposed NAH is useful as an alternative to the conventional NAH method.

\section{Conclusion}

Experiments with the proposed NAH method are performed. As a result, the proposed method is shown to be a useful alternative to conventional NAH. It is also shown that the proposed NAH can considerably reduce the workload of measurement to the same level as that when using AI for high frequencies.

By using the proposed NAH with extra spatialdomain spectrums, Sound fields can be measured with the same workload and same equipment as those when using AI for high-frequency sound fields.

\section{References}

( 1 ) Nagamatsu, M., A Research about NAH Method with k-Domain Odd Spectrum Lines, Internoise 2002 Book of Abstracts, (2002), p.28.

(2) Nagamatsu, M., A Theoretical Argument of NAH Method with Extra k-Domain Spectrums, Internoise 2003 Book of Abstracts, (2003), p.129.

( 3 ) Maynard, J.D., Williams, E.G. and Lee, Y., Nearfield Acoustic Holography: I. Theory of Generalized Holography and the Development of NAH, J. Acoust. Soc. Am., Vol.78 (1985), pp.1395-1413.

( 4 ) Veronesi, W.A. and Maynard, J.D., Nearfield Acoustic Holography (NAH) II. Holographic Reconstruction Algorithms and Computer Implementation, J. Acoust. Soc. Am., Vol.81 (1987), pp.1307-1322.

( 5 ) Hald, J., Combined NAH and Beamforming Using the Same Array, Technical Review 2005, No.1 (2005), pp.11-39

( 6 ) Williams, E.G., Fourier Acoustics - Sound Radiation and Nearfield Acoustical Holography, (1999), Academic Press, San Diego. 